\title{
ASPECTOS NEUROLÓGICOS E ELETRENCEFALOGRÁFICOS DA TOXOPLASMOSE
}

\author{
ADHEMar M. Fiorillo * \\ Jorge ARMBRUST Frgueiredo** \\ Rubens Moura Ribeiro ***
}

Desde 1937, quando Wolf e Cowen ${ }^{18}$ demonstraram a ocorrência da toxoplasmose na espécie humana, numeroso grupo de investigadores, constituido principalmente por obstetras e pediatras, passou a se interessar pelo problema, em razão de natureza predominante congênita da moléstia. Entretanto, a forma tardia da toxoplasmose com acometimento visceral, muscular, ocular e do sistema nervoso, evidencia em geral um quadro clínico discreto ou, na maioria das vêzes, assintomático $1,12,15$. No que diz respeito ao sistema nervoso, a dificuldade do diagnóstico da toxoplasmose, decorrente da impossibilidade de se demonstrar in vivo o comprometimento cerebral dos pacientes, torna necessária a utilização dos exames subsidiários com intuito de obter apoio mais objetivo à suspeita clínica.

A oportunidade que tivemos de observar os aspectos neurológicos em um grupo de pacientes com toxoplasmose, permitiu-nos realizar um estudo descritivo e assinalar de maneira objetiva as alteraçōes verificadas.

\section{MATERIAL E MÉTODOS}

O material consta de 30 pacientes com toxoplasmose, comprovada pela presença de coriorretinjte e pela positividade da reaçāo Sabin-Feldman. No quadro 1 figuram os dados relativos à identidade, sexo e idade dos pacientes, assim como os resultados da reacāo de Sabin-Feldman cujos títulos variaram de 1/64 até 1/8000.

Ccmplementando os exames clinicos e neurológicos foram feitas radiografias do tórax e do crânio, exame do líquido cefalorraqueano e eletrencefalograma. As reações sorológicas de Wassermann, de Machado Guerreiro e de fixaçāo de complemento para brucelose foram negativas em todos os casos.

\section{RESULTADOS}

O exame de líquido cefalorraqueano foi negativo em todos os casos. o exame radiológico mostrou calcificaçöes intracranianas em 3 (casos 1,2 e 7 ). 0 exame neurológico nada mostrou em 25 casos; em 4 casos (8, 11, 16 e 24) havia estrabis-

Trabalho da Clinica Neurológica (Prof. J. Armbrust Figueiredo) e da Clinica Médica (Prof. Hélio Lourenço de Oliveira) da Fac. Med. de Ribeirão Prêto da Univ. de São Paulo: * Assistente-Docente; ** Professor; *** Assistente. 
mo e, em um (caso 4), foi assinalada diminuição do reflexo corneano à esquerda. Nenhum paciente apresentava crises convulsivas. Entretanto, em 22 casos, ou seja $73,4 \%$, foram registradas alteraçôes eletrencefalográficas, distribuídas da seguinte forma: disritmia paroxistica ( 14 casos, ou seja $46,6 \%$ ); desorganização do ritmo (6 casos, ou seja 20\%); assimetria de ritmo (5 casos, ou seja 16,6\%). A soma dos resultados eletrencefalográficos parcelados ultrapassa o valor total em virtucie de um mesmo traçado evidenciar mais de um tipo de alteração.

\section{COMENTARIOS}

Analisando os resultados dos exames neurológicos e liquóricos, verificamos que pràticamente sāo nulos os dados patológicos. Por outro lado, os registros eletrencefalográficos mostraram alteraçōes em $73,4 \%$ dos casos, percentagem bem maior que a usual em população normal 4, 5, 13.

Portanto, o EEG dos pacientes com toxoplasmose é um métodu semiológico útil, já que os achados neurológicos são bastante precários e o estudo radiológico do crânio nem sempre mostra calcificaçōes cerebrais patológicas".

Caso Nome Registro Sexo Idade (anos) Sabin-Feldman

\begin{tabular}{rlrlll}
\hline \hline 1 & CAR & 18673 & M & 25 & \\
2 & JMF & 7602 & M & 37 & $1 / 1000$ \\
3 & IB & 10526 & F & 28 & $1 / 256$ \\
4 & EF & 12732 & M & 39 & $1 / 1000$ \\
5 & AGB & 13886 & F & 46 & $1 / 256$ \\
6 & SF & 16882 & M & 22 & $1 / 256$ \\
7 & MLCP & 15417 & F & 49 & $1 / 64$ \\
8 & GM & 10744 & M & 20 & $1 / 1000$ \\
9 & GMS & 23007 & F & 24 & $1 / 256$ \\
10 & MAM & 9030 & F & 26 & $1 / 256$ \\
11 & GAP & 21252 & M & 7 & $1 / 256$ \\
12 & DGS & 2050 & F & 23 & $1 / 256$ \\
13 & AM & 23063 & M & 23 & $1 / 1000$ \\
14 & GMR & 24305 & F & 15 & $1 / 1000$ \\
15 & LC & 23538 & M & 30 & $1 / 1000$ \\
16 & OD & 1388 & F & 26 & $1 / 64$ \\
17 & NANA & 15891 & M & 30 & $1 / 1000$ \\
18 & JMF & 23003 & F & 42 & $1 / 64$ \\
19 & LTS & 12498 & M & 43 & $1 / 1000$ \\
20 & RFTS & 12497 & F & 10 & $1 / 1000$ \\
21 & RATS & 12176 & F & 14 & $1 / 1000$ \\
22 & RMTS & 10820 & M & 12 & $1 / 256$ \\
23 & ORN & 26369 & M & 21 & $1 / 256$ \\
24 & ABS & 26707 & M & 5 & $1 / 8000$ \\
25 & JP & 15060 & M & 33 & $1 / 256$ \\
26 & WC & 9680 & F & 25 & $1 / 4000$ \\
27 & NT & 28714 & M & 28 & $1 / 256$ \\
28 & RR & 13974 & M & 47 & $1 / 64$ \\
29 & LCCV & 29522 & M & 8 & $1 / 4000$ \\
30 & JPG & 9122 & F & 39 & $1 / 1000$ \\
& & & & & \\
\hline
\end{tabular}

Quadro 1 - Resultados da reaçio de Sabin-Feldman em 30 casos examinados. 
Considerando apenas os resultados eletrencefalográficos, sāo evidentes os sinais de comprometimento cerebral observados em nossos pacientes em virtude da alta incidência de alteração no ritmo de base.

Comparando nossos achados com os de Thiry ${ }^{16}$, Lelong e col. ${ }^{8}$, que analisaram os EEG de pacientes portadores de toxoplasmose adquirida, registramos as mesmas alteraçōes assinaladas por aquêles autores e que são sugestivas de processo lesional cerebral. Portanto, a presença de disritmia pai ixística e de desorganização do ritmo de base em elevada percentagem em nossos pacientes, poderia constituir a única seqüela de um quadro meningo-encefalítico tão comum nesses pacientes $3,6,7,9,10,11,14,17,19$.

Os estudos eletrencefalográficos nos pacientes com toxoplasmose são ainda pouco numerosos e é nosso intuito valorizar oportunamente a utilidade do registro eletrencefalográfico nos casos com reação de Sabin-Feldman significativamente positiva.

\section{RESUMO}

Foram estudados do ponto de vista neurológico 30 pacientes portadores de coriorretinite e com reaçāo de Sabin-Feldman positiva para toxoplasmose: não foram encontradas alteraçōes de importância nos exames neurológicos; em três casos o exame radiológico mostrou calcificações intracranianas; em todos os casos foi normal o exame de liquido cefalorraqueano.

A análise dos registros eletrencefalográficos evidenciou, entretanto, a presença de anormalidades em $73,4 \%$ dos casos (disritmias paroxisticas, desorganização do ritmo de base e assimetria entre a atividade elétrica dos hemisférios cerebrais). O EEG é, portanto, um método útil para a demonstração de alterações cerebrais nos pacientes com toxoplasmose.

\section{SUMMARY}

Neurologic and electroencephalographic aspects of toxoplasmosis.

Thirty patients with chorioretinitis and positive Sabin-Feldman dye test were studied concerning their neurologic aspects. There were no striking abnormalities in neurologic examination and skull $\mathrm{X}$-rays. Otherwise electroencephalographic studies indicate that $73.4 \%$ of the 30 patients showed some degree of abnormalities. The most frequent changes consisted of paroxysmal sharp waves patterns, diffuse desorganization changes and assymetrical brain waves activities. It should be emphasized the high incidence of electroencephalographic abnormalities in patients with toxoplasmosis.

\section{REFERE̊NCIAS}

1. ARRIAGADA, C.; BECHAR, V. e MARTINEZ, A. - Neurotoxoplasmosis adquirida en adultos. Comunicación de dos casos verificados por necropsia y revisión 
de algunas caracteristicas de la afección. Acta Neurol. Latinoamer, 6:257-288, 1960. 2. DYKE, C. G.; WOLF, A.; COWEN, D.; PAIGE, B. H. e CAFFEY, J. - Toxoplasmic encephalomyelitis: VIIr. Significance of roentgenographic findings in the diagnosis of infantile or congenital toxoplasmosis. Am. J. Roentgenol., 47:830-841, 1962. 3. EICKE-WERNER, J. - Toxoplasmose Enzephalitis. Nervenarzt 25:387-388, 1954. 4. GIBBS, F. A.; GIBBS, E. L. e LENNOX, W. C. - Electroencephalographic classification of epileptic patients and control subjects. Arch. Neurol. a. Psychiat., 50: 111-128, 1943. 5. GIBBS, F. A.; GIBBS, E. L. e LENNOX, W. C. - Electroencephalographic response to overventilation and its relation age. J. Pediat., 23:497-505, 1\$43. 6. HAFSTROM, T. - Toxoplasmic encephalopathy: a form of meningo-encephalo-myelitis in adult toxoplasmosis. Acta Psychiat. et Neurol. Scandinav., 34: 310-321, 1959. 7. KRESTSCHMER, W. e SCHMIDT, E. - Komplizierte psychose bei toxoplasmosis enzephalitis. Arch. Psychiat., 193:38-47, 1955. 8. LELONG, M.; BERNARD, J.; DEMONTS, G. e COUVREUR, J. - La toxoplasmose acquise: étude de 227 observations. Arch. Franç. Pédiat., 17:281-331, 1960. 9. PAINE, R. S. - Emergencies of cerebral origin. Pediat. Clin. N. Amer., 9:87, 1962. 10. PILLERI, G. Beitrag zur pathologie der toxoplasma-enzephalitjs. Monatsschr. f. Psychiat. u. Neurol., 127:250-272, 1954. 11. REMINGTON, J. S.; JACOBS, L. e KAUFMAN, H. E. - Toxoplasmosis in the adult. New England J. Med., 262:180-186, 1960. 12. SABIN, A. B. - Toxoplasmic encephalitis in children. JAMA, 116:801-807, 1941. 13. SCHWAB, R. S. - Electroencephalography in Clinical Practice. W. B. Saunders Co., Philadelphia, 1951. 14. SEXTON, R. C.; EYLES, D. E. e DILLMAN, R. E. - Aduit toxoplasmosis. Medicine 14:366-377, 1953. 15. SIIM, J. C. - Acquired toxoplasmosis. Report of seven cases with strongly positive sorologic reactions. JAMA, 147:16411645, 1951. 16. THIRY, S. - Etude électroclinique d'un cas de toxoplasmose. Acta Neurol. et Psychiat. Belg., 53:230-233, 1953. 17. WETTINGFELD, R. F.; ROWE, J. e EYLES, D. E. - Treatment of toxoplasmosis with pyrimethamine (Daraprim) and triplesulfonamide. Ann. Inst. Med., 44:557-574, 1956. 18. WOLF, A. e COWEN Jr., D. - Granulomatose encephalomyelitis due to an Encephalitozoon (Encephalitozoje encephalomyelitis); new protozoon disease of man. Bull. Neurol. Inst. New York 6:306-371, 1939. 19. WOLHEIM, E. - Zur Klinük der Erwachsenen Toxoplasmose. München. med. Wchnschr., 94:194-199, 1952.

Clinica Neurológica, Faculdade de Medicina - Ribeirüo Prêto, São Paulo - Brasil. 